\title{
Editorial
}

Neuroepidemiology

\section{The Evolution of Neuroepidemiology: Marking the 40-Year Anniversary of Publishing Studies on Epidemiology of Neurological Disorders}

\author{
Valery L. Feigin \\ National Institute for Stroke and Applied Neurosciences, Auckland University of Technology, \\ Auckland, New Zealand
}

The critical importance of epidemiological studies is particularly evident at the time of unexpected pandemics, such as COVID-19, which has multiple neurological complications. According to the recent WHO reports, the global death toll of COVID-19 is already more than 5.2 million and continues to increase. Every government and national and local policymakers are aware of the situation in their regions and follow the health statistics to accommodate the needs of the patients and plan health care and resource allocation accordingly to reduce the impact of the pandemic. In addition, thousands of researchers worldwide and even whole research institutions and laboratories are exploring ways to treat and prevent the disease to eventually stop the pandemic.

As a neurologist and clinical epidemiologist, I would like to see the same attention paid by the governments, health care policymakers, funders, and other stakeholders to the even greater pandemic of neurological disorders. According to the most recent Global Burden of Disease (GBD) Study, neurological disorders are currently the leading cause of disability and the second leading cause of death in the world. Their burden over the last 30 years is increasing fast [1]. When the journal Neuroepidemiology was first launched in 1982, there was no reliable global

karger@karger.com

(c) 2021 S. Karger AG, Basel

www.karger.com/ned

Karger data on the burden of neurological disorders. The first reliable data on the global, regional, and national burden of neurological disorders (incidence, prevalence, mortality, years lived with disability, years of life lost, and disability-adjusted life-years lost [DALYs]) by age, sex, and sociodemographic index and/or country income level came from the GBD Study. These data are updated annually and now even include subnational estimates for some countries (e.g., USA, UK, Brazil, Italy, China) as the scientific basis for health care planning, resource allocation, and public health priority setting. According to the most recent GBD estimates, one in three people in the world is now suffering from a neurological disorder ranging from migraine to stroke and dementia [1], and the total number of people who died from neurological disorders over the last three decades has increased by $61 \%$ (from 5.5 million in 1990 to 8.8 million in 2019) [2], with the five largest contributors to global neurological DALYs being stroke $(42.2 \%)$, migraine (16.3\%), dementia (10.4\%), meningitis (7.9\%), and epilepsy (5.0\%). Almost $80 \%$ of deaths and DALYs due to neurological disorders reside in low- and middle-income countries. Just in the USA and Europe alone, the annual cost of neurological disorders amounts to USD 1.7 trillion [3, 4], which is almost two 
times the estimated global cost of cardiovascular disease, including stroke (USD 957 billion) [5]. The already massive burden of neurological (brain) disorders is likely to increase significantly in the near future due to the ongoing aging and growth of the population across the globe.

Over the last 40 years, huge progress has been made in the understanding of etiology, determinants, outcomes, and management of many neurological disorders. For example, from being a largely neglected, nonpreventable, and nontreatable disease listed in all previous International Classifications of Diseases (ICD) under cardiovascular disorders, stroke is now a well-recognized, highly preventable, and highly treatable disease, rightfully placed in the ICD-11 under the rubric of neurological disorders. Significant advancements have been made in the epidemiology and understanding of inter-relationships of vascular and Alzheimer's type of dementia and dementia prevention, evaluation of the impact of environmental (e.g., ambient air pollution), metabolic, socioeconomic, and lifestyle factors on the risk of neurological disorders in different regions and populations $[1,6,7]$, and ethnic/ racial disparities in the epidemiology of and care for various neurological disorders [8]. More recently, we are also witnessing significant progress in neurogenetics [9], precision medicine [10], digital technology, big data, machine learning, and artificial intelligence in the diagnosis, prognosis, and prevention of some neurological disorders $[11,12]$. All these scientific achievements in the epidemiology, prevention and management of neurological disorders, along with the already huge and fast-growing psychological, physical, mental, and economic burden of neurological disorders on individuals, families, and societies have led the UN, WHO, and other large international organizations to develop a range of unprecedented measures to stop and eventually reverse the burden of neurological disorders in the world.

By publishing high-quality studies on the frequency, distribution, and determinants of neurological disorders in humans over the last 40 years, our international journal Neuroepidemiology contributes its share to the global fight against neurological disorders. However, the fight has just begun and, as the Editor-in-Chief of the journal, I call upon our readers, neurologists, public health physicians, and all other health professionals and experts involved in the fight against neurological disorders to continue submitting their research manuscripts to the journal to further advance our knowledge in the epidemiology of neurological disorders around the globe. Finally, I would like to thank all our journal authors, reviewers, co-editors, Editorial Board members, and Karger Publisher staff for their ongoing support to the journal over the years and congratulate them all with the 40-year anniversary of Neuroepidemiology.

\section{Conflict of Interest Statement}

There is no conflict of interest to disclose.

\section{Funding Sources}

There were no funding sources for this work.

\section{References}

1 GBD 2016 Neurology Collaborators. Global, regional, and national burden of neurological disorders, 1990-2016: a systematic analysis for the Global Burden of Disease Study 2016. Lancet Neurol. 2019;18:459-80.

2 Institute for Health Metrics and Evaluation (IHME). GBD compare data visualization. Seattle, WA: IHME, University of Washington; 2019. Available from: https://vizhub. healthdata.org/gbd-compare/ Accessed 2021 Nov 9.

3 Gooch CL, Pracht E, Borenstein AR. The burden of neurological disease in the United States: a summary report and call to action. Ann Neurol. 2017;81:479-84.

4 Olesen J. The cost of neurological disorders in Europe. J Neurol Sci. 2015;357:e500-1.
5 Driving sustainable action for circulatory health. White paper on circulatory health. Geneva, Switzerland. Global coalition for circulatory health; 2018. Available from: https:// world-heart-federation.org/resource/whitepaper-for-circulatory-health/ Accessed 2021 Nov 24.

6 Livingston G, Huntley J, Sommerlad A, Ames D, Ballard C, Banerjee S, et al. Dementia prevention, intervention, and care: 2020 report of the Lancet Commission. Lancet. 2020;396: 413-46.

7 Brainin M, Feigin VL, Norrving B, Martins SCO, Hankey GJ, Hachinski V. Global prevention of stroke and dementia: the WSO Declaration. Lancet Neurol. 2020;19:487-8.

8 The Lancet Neurology. Disparities in neurological care: time to act on inequalities. Lancet Neurol. 2020;19:635.
9 Zamba-Papanicolaou E. Practical guide to neurogenetics. Lancet Neurol. 2009;8(6):516.

10 Caskey CT, Posey JE, Hou Y-CC, Yu H-C. Precision medicine in neurology. In: Rosenberg RN, Pascual JM, editors. Rosenberg's molecular and genetic basis of neurological and psychiatric disease. 6th ed. Cambridge, MA: Academic Press; 2020. p. 27-39.

11 Moccia M, Brigo F, Brennan S, Bonavita S. Editorial: digital technology in neurology: from clinical assessment to neurorehabilitation. Front Neurol. 2020;11:614074.

12 Auger SD, Jacobs BM, Dobson R, Marshall CR, Noyce AJ. Big data, machine learning and artificial intelligence: a neurologist's guide. Pract Neurol. 2021;21:4-11. 